\title{
Breakfast in relation to overall diet quality in UK children and adolescents
}

\author{
S. Gaal, M.B.E. Livingstone, H. McNulty, M. Ward and M.A. Kerr \\ Nutrition Innovation Centre for Food and Health (NICHE), School of Biomedical Sciences, Ulster University, \\ Coleraine, BT52 ISA.
}

Evidence suggests that regular breakfast consumption is associated with higher overall micronutrient intake $\mathrm{e}^{(1)}$, dietary adequacy ${ }^{(2)}$ and quality $^{(3)}$ in children and adolescents but this has not been fully explored in a UK cohort ${ }^{(4)}$. The objective of this study was therefore to investigate the relationship between breakfast and overall diet quality in UK children and adolescents aged 5-17 years. Data from the most recent National Diet and Nutrition Survey (NDNS, 2008-2014) were accessed to provide a representative sample of UK children and adolescents $(n=3283)$. Dietary intake was assessed by a 4-day estimated food diary. As meal occasions were not specified in NDNS, breakfast was defined for the current study as all food and drink items consumed between 6 am - 11 am. Average energy, macro- and micronutrient intakes from breakfast were calculated based on consumption days only. Overall diet quality (DQ) was assessed by applying the Nutrient Rich Food Index (NRF) 9.3 method $^{(5)}$ which subtracts the sum of three negative nutrients (saturated fat, added sugar and salt) from that of nine positive nutrients (protein, fibre, vitamin A, C, D, calcium, iron, potassium, magnesium) expressed as a multiple of $100\left(\sum\right.$ sub-scores positive $\left.\times 100\right)-\left(\sum\right.$ sub-scores negative $\left.\times 100\right)$. The algorithm was adjusted based on an 8.4 MJ energy intake. Statistical analyses were carried out by SPSS (Version 24) software.

Table 1.

\begin{tabular}{|c|c|c|c|c|c|c|c|c|}
\hline \multirow[b]{3}{*}{ Breakfast intakes } & \multicolumn{6}{|c|}{ Children $(5-17$ years $)(n=3283)$} & \multirow[b]{3}{*}{$\mathrm{P}$ value* } & \multirow[b]{3}{*}{$\mathrm{P}$ value** } \\
\hline & \multicolumn{2}{|c|}{ T1 $(n=1094)$} & \multicolumn{2}{|c|}{ T2 $(n=1095)$} & \multicolumn{2}{|c|}{ T3 $(n=1094)$} & & \\
\hline & Mean & $\mathrm{SD}$ & Mean & $\mathrm{SD}$ & Mean & $\mathrm{SD}$ & & \\
\hline NRF 9.3 Score & 483 & 68 & 608 & 26 & 717 & 49 & $<0.001$ & $<0.001$ \\
\hline Energy (kJ)¥ & 1518 & 738 & 1452 & 615 & 1439 & 575 & $0 \cdot 156$ & $0 \cdot 113$ \\
\hline AOAC fibre $(\mathrm{g}) ¥$ & $2 \cdot 5$ & 1.9 & 3 & $1 \cdot 8$ & 3.7 & $2 \cdot 2$ & $<0 \cdot 001$ & $<0 \cdot 001$ \\
\hline Total fat (\% energy)¥ & 29 & 11 & 28 & 10 & 25 & 10 & $<0 \cdot 001$ & $<0 \cdot 001$ \\
\hline Total SFA ( $\%$ energy)¥ & 13 & 6 & 12 & 5 & 11 & 4 & $<0 \cdot 001$ & $<0 \cdot 001$ \\
\hline Total sugars (\% energy)¥ & 32 & 15 & 30 & 13 & 29 & 12 & $<0 \cdot 001$ & $<0 \cdot 001$ \\
\hline NMES (\% energy) $¥$ & 23 & 15 & 18 & 12 & 14 & 9 & $<0.001$ & $<0.001$ \\
\hline Folate $(\mu \mathrm{g}) ¥$ & 56 & 39 & 67 & 40 & 79 & 44 & $<0.001$ & $<0.001$ \\
\hline Calcium (mg)¥ & 226 & 140 & 244 & 145 & 260 & 142 & $<0.001$ & $<0.001$ \\
\hline Iodine $(\mu \mathrm{g}) ¥$ & 35 & 28 & 41 & 29 & 48 & 34 & $<0 \cdot 001$ & $<0.001$ \\
\hline Iron (mg)¥ & $2 \cdot 5$ & $1 \cdot 6$ & 2.9 & $1 \cdot 7$ & $3 \cdot 3$ & $1 \cdot 8$ & $<0.001$ & $<0.001$ \\
\hline Magnesium (mg)¥ & 41 & 22 & 46 & 23 & 53 & 26 & $<0 \cdot 001$ & $<0.001$ \\
\hline Potassium (mg)¥ & 419 & 231 & 470 & 230 & 523 & 243 & $<0 \cdot 001$ & $<0 \cdot 001$ \\
\hline Sodium (mg)¥ & 420 & 338 & 378 & 263 & 356 & 219 & $<0.001$ & $0 \cdot 001$ \\
\hline
\end{tabular}

Tertile 1: Low DQ; Tertile 2: Medium DQ; Tertile 3: High DQ. P* unadjusted (ANOVA) P** adjusted for National Statistics Socio-economic Classification (housing, employment, education) (ANCOVA). ¥ Nutrient values were Square-root transformed for normalisation purposes prior to analysis; data shown as absolute values. Macronutrients expressed as percentage contribution to breakfast energy. P $<0.05$ is considered significant. Abbreviations: AOAC American Association of Analytical Chemists; SFA saturated fat; NMES non-milk extrinsic sugars.

Table 1 shows dietary intake at breakfast according to tertiles of NRF score. Average breakfast energy intake did not differ by tertile of DQ. However, average breakfast intakes of fibre, folate, calcium, iodine, iron, magnesium and potassium progressively and significantly increased from the lowest to the highest tertile of DQ. In contrast, average breakfast intakes of total fat, saturated fat, added sugar and sodium showed the opposite trend. These results highlight an important role for breakfast in relation to overall diet quality. The development of nutrient based breakfast recommendations for children and adolescents may be warranted.

This study was funded by Cereal Partners Worldwide as part of the International Breakfast Research Initiative.

1. Coulthard JD, Palla L \& Pot GK (2017) Br J Nutr 118, 280-90.

2. Barr SI, DiFrancesco L \& Fulgoni VL 3rd. (2014) Br J Nutr 112, 1373-83.

3. Polonsky HM, Davey A, Bauer KW et al. (2018) J Nutr Educ Behav 50, 190-197.

4. Llaurado E, Albar SA, Giralt M et al. (2016) Eur J Nutr 55, 1789-97.

5. Maillot M, Ferguson EL, Drewnowski A et al. (2008) J Nutr 138, 1107-13. 